\title{
Gaze-Contingent Ocular Parallax Rendering for Virtual Reality
}

\author{
ROBERT KONRAD, ANASTASIOS ANGELOPOULOS, and GORDON WETZSTEIN, Stanford University
}
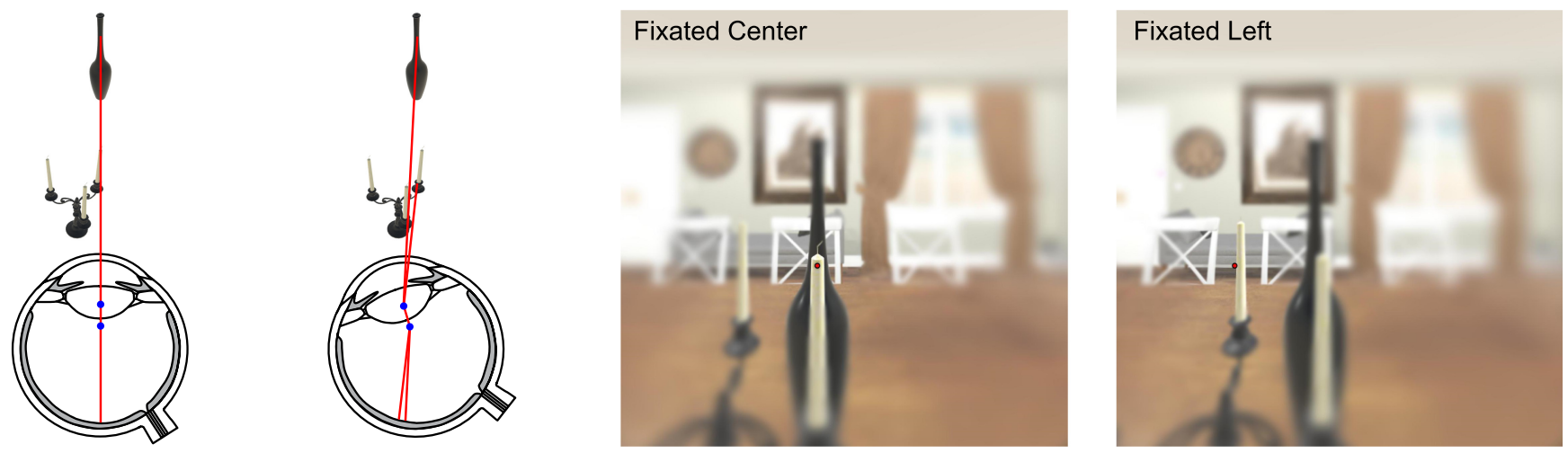

Fig. 1. The centers of rotation and projection of the eyes are not the same. As a consequence, small amounts of parallax are created in the retinal image as we fixate on different objects in the scene. The nodal points of the eye, representing the centers of projection, are shown as small blue circles on the left along with a ray diagram illustrating the optical mechanism of ocular parallax. Simulated retinal images that include the falloff of acuity in the periphery of the visual field are shown on the right. As a user fixates on the candle in the center of the scene (center, red circle indicates fixation point), the bottle is partly occluded by the candle. As their gaze moves to the left, ocular parallax reveals the bottle behind the candle in the center (right). Ocular parallax is a gaze-contingent effect exhibiting the strongest effect size in near to mid peripheral vision, where visual acuity is lower than in the fovea. In this article, we introduce ocular parallax rendering for eye-tracking-enabled virtual reality displays and study the complex interplay between micro parallax, occlusion, visual acuity, and other perceptual aspects of this technology in simulation and with a series of user experiments.

Immersive computer graphics systems strive to generate perceptually realistic user experiences. Current-generation virtual reality (VR) displays are successful in accurately rendering many perceptually important effects, including perspective, disparity, motion parallax, and other depth cues. In this article, we introduce ocular parallax rendering, a technology that accurately renders small amounts of gaze-contingent parallax capable of improving depth perception and realism in VR. Ocular parallax describes the small amounts of depth-dependent image shifts on the retina that are created as the eye rotates. The effect occurs because the centers of rotation and projection of the eye are not the same. We study the perceptual implications of ocular parallax rendering by designing and conducting a series of user experiments. Specifically, we estimate perceptual detection and discrimination thresholds for this effect and demonstrate that it is clearly visible in most VR applications. Additionally, we show that ocular parallax rendering provides an effective ordinal depth cue and it improves the impression of realistic depth in VR.

This project was generously supported by funding from the National Science Foundation (NSF, award numbers 1553333 and 1839974), a Sloan Fellowship, an Okawa Research Grant, and Intel.

Authors' addresses: R. Konrad, A. Angelopoulos, and G. Wetzstein, Stanford University; emails: rkkonrad@stanford.edu; nikolasa@stanford.edu; gordon. wetzstein@stanford.edu.

Permission to make digital or hard copies of all or part of this work for personal or classroom use is granted without fee provided that copies are not made or distributed for profit or commercial advantage and that copies bear this notice and the full citation on the first page. Copyrights for components of this work owned by others than the author(s) must be honored. Abstracting with credit is permitted. To copy otherwise, or republish, to post on servers or to redistribute to lists, requires prior specific permission and/or a fee. Request permissions from permissions@acm.org.

(C) 2020 Copyright held by the owner/author(s). Publication rights licensed to ACM.

0730-0301/2020/01-ART10 \$15.00

https://doi.org/10.1145/3361330
CCS Concepts: • Computing methodologies $\rightarrow$ Mixed / augmented reality; Perception; Virtual reality;

Additional Key Words and Phrases: Computational displays, virtual reality, augmented reality, eye tracking, gaze-contingent rendering

\section{ACM Reference format:}

Robert Konrad, Anastasios Angelopoulos, and Gordon Wetzstein. 2020. Gaze-Contingent Ocular Parallax Rendering for Virtual Reality. ACM Trans. Graph. 39, 2, Article 10 (January 2020), 12 pages.

https://doi.org/10.1145/3361330

\section{INTRODUCTION}

Immersive computer graphics systems, such as virtual reality (VR) displays, aim at synthesizing a perceptually realistic user experience. To achieve this goal, several components are required: interactive, photorealistic rendering; a high-resolution, lowpersistence, stereoscopic display; and low-latency head tracking. Modern VR systems provide all of these capabilities and create experiences that support many, but not all, of the monocular and binocular depth cues of the human visual system, including occlusions, shading, binocular disparity, and motion parallax. The support of focus cues (e.g., accommodation and retinal blur) has also received attention in research and industry over the last few years. In this article, we study a depth cue of human vision that has not been discussed in the context of virtual reality and that may help further improve depth perception and perceptual realism: ocular parallax.

The centers of rotation and projection in the human eye are not the same. Therefore, changes in gaze direction create small 
amounts of depth-dependent image shifts on our retina-an effect known as ocular parallax. This depth cue was first described by Brewster [1845] and it has been demonstrated to produce parallax well within the range of human visual acuity [Bingham 1993; Hadani et al. 1980; Mapp and Ono 1986]. Interestingly, species as diverse as the chameleon and the sandlance critically rely on this depth cue to judge distance [Land 1995; Pettigrew et al. 1999].

To render ocular parallax into a VR/augmented reality (AR) experience, eye tracking is required. Conveniently, many emerging wearable display systems already have eye tracking integrated, either to support foveated rendering [Guenter et al. 2012; Patney et al. 2016], accurate registration of physical and digital images in $\mathrm{AR}$, or other gaze-contingent display modes. With eye tracking available, there is no additional computational cost to integrate ocular parallax. The perspective of the rendered image simply changes depending on the gaze direction. However, the magnitude of depth-dependent motion induced by ocular parallax rendering increases in the periphery of the visual field, where visual acuity is lower than in the fovea. Moreover, the resolution offered by current-generation VR displays is well below the visual acuity of human vision and it is not clear if the subtle ocular parallax effect is perceivable in VR at all.

To further our understanding of ocular parallax and its perceptual effects in VR, we thoroughly analyze the tradeoffs between perceived parallax, visual acuity, and disparity for near-eye displays. We build a prototype gaze-tracked VR display, conduct a series of user experiments that quantify effect sizes of ocular parallax rendering, and measure its impact on depth perception and the user experience in general. We find that detection thresholds for ocular parallax rendering are almost an order of magnitude lower than the visual acuity at the same extrafoveal locus, which verifies that our sensitivity to small amounts of differential motion is well below the acuity limit, especially in the periphery of the visual field [Mckee and Nakayama 1984]. We also show that the relative ocular parallax of objects with respect to a background target can be discriminated accurately even for relatively small object distances that fall well within the depth ranges of most virtual environments. Furthermore, we show that ocular parallax rendering provides an effective ordinal depth cue, helping users better distinguish the relative depth ordering of a scene, but that it does not necessarily benefit absolute, or metrical, distance estimates to objects. Finally, we show that ocular parallax rendering improves the impression of realistic depth in a 3D scene. For no additional computational cost, ocular parallax rendering has the potential to improve both depth perception and perceptual realism of eye-tracked AR/VR systems.

Specifically, we make the following contributions:

- We introduce gaze-contingent ocular parallax rendering for VR display systems.

- We design and conduct user experiments to quantify detection and discrimination thresholds of ocular parallax rendering.

-We design and conduct user experiments to quantify the effectiveness of ocular parallax rendering as both an ordinal and absolute depth cue.

- We conduct a user experiment that demonstrates improved perceptual realism using ocular parallax rendering.

\section{RELATED WORK}

Depth Cues. Human depth perception relies on a variety of cues [Howard and Rogers 2002; Palmer 1999]. Many of these cues are pictorial and can be synthesized using photorealistic rendering techniques, including occlusions, perspective foreshortening, texture and shading gradients, as well as relative and familiar object size. Unlike conventional $2 \mathrm{D}$ displays, head-mounted displays (HMDs) use stereoscopic displays and head tracking and can thus support two additional visual depth cues, disparity and motion parallax, as well as one oculomotor cue, vergence. Emerging neareye displays also support visual focus cues like retinal blur and chromatic aberrations, which in turn drive accommodation, another oculomotor cue (see discussion below). All of these cues are important for human depth perception to varying degrees depending on the fixation distance [Cutting and Vishton 1995]. Studying visual cues, such as disparity [Didyk et al. 2011] or motion parallax [Kellnhofer et al. 2016], and their impact on computational display applications has been an integral part of graphics research. In this work, we explore ocular parallax as another visual cue that may improve the user experience in immersive computer graphics applications.

Ocular Parallax. Ocular parallax describes the change in perspective as the eye rotates, primarily due to the user fixating on different parts of the scene. This visual cue is well known [Brewster 1845] and has a measurable effect on depth perception [Bingham 1993; Kudo and Ohnishi 1998; Kudo et al. 1999; Mapp and Ono 1986]. Similar to other monocular visual cues, such as retinal blur and chromatic aberration, the change of the retinal image caused by ocular parallax may be small. Nonetheless, supporting all of these cues with an HMD can improve visual comfort [Hoffman et al. 2008], perceived realism, and the user experience as a whole.

Kudo and Ohnishi [2000] discuss gaze-contingent optical distortions in HMDs and attribute them in part to ocular parallax. This effect is commonly known as "pupil swim." However, they did not propose ocular parallax rendering for HMDs or study its perceptual effects with HMDs. Building on emerging HMDs with eyetracking technology, to our knowledge, we are the first to propose ocular parallax as a gaze-contingent rendering mode for VR/AR and evaluate its perceptual implications with a series of user experiments.

Gaze-contingent and Computational Displays. Eye tracking enables gaze-contingent rendering techniques that adapt effects like magnification, stylization, or geometric level-of-detail to the user's viewpoint [Duchowski et al. 2004]. Gaze-contingent rendering is becoming an integral part of modern near-eye display systems, enabling techniques such as foveated rendering [Guenter et al. 2012; Patney et al. 2016], and gaze-contingent varifocal [Dunn et al. 2017; Johnson et al. 2016; Konrad et al. 2015; Liu et al. 2008; Padmanaban et al. 2017] or multifocal [Akeley et al. 2004; Mercier et al. 2017; Rolland et al. 2000] displays. Although rendering accommodationdependent effects, such as chromatic aberrations [Cholewiak et al. 2017] and blur at depth edges [Marshall et al. 1996; Zannoli et al. 2014], have not been directly evaluated with eye-tracked displays, these techniques could be optimized by tracking the user's gaze or accommodation. Ocular parallax rendering is complimentary to 


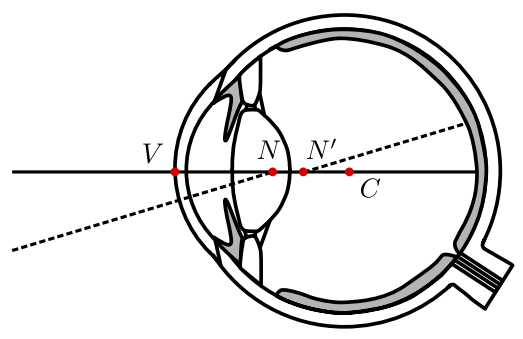

Fig. 2. Illustration of a schematic eye, including the front and rear nodal points $N, N^{\prime}$, the center of rotation $C$, and the anterior vertex of the cornea $V$. The nodal points are two parameters of a thick lens model that refract light rays as depicted. The exact locations of these points depend on the schematic eye model used.

these techniques and could be integrated, without computational overhead, into conventional HMDs with eye tracking and optionally combined with other gaze-contingent rendering algorithms.

Other than the proposed method, the only techniques described in the literature that inherently provide ocular parallax cues are near-eye multifocal displays [Hu and Hua 2014; Llull et al. 2015; Love et al. 2009; Narain et al. 2015], light field displays [Hua and Javidi 2014; Huang et al. 2015; Lanman and Luebke 2013], and holographic displays [Maimone et al. 2017; Padmanaban et al. 2019]. However, the effect of ocular parallax or lack thereof has not been investigated in any of the aforementioned technologies. In fact, ocular parallax in multifocal displays is often undesirable because it reveals misalignments between the virtual image planes; Mercier et al. [2017] proposed a multifocal display that effectively removes the ocular parallax cue by shifting the decomposed layers according to the tracked pupil position.

\section{OCULAR PARALLAX}

In this section, we discuss schematic models of the human eye with a specific focus on how they model the centers of projection and rotation. Moreover, we discuss and analyze the tradeoff between ocular parallax and image sharpness, accounting for the decrease in peripheral visual acuity as well as retinal blur due to accommodation.

Eye Models. Complex optical systems with multiple refractive surfaces can be reduced to six cardinal points that fully define the Gaussian imaging and magnification properties of the system. These cardinal points include the front and rear focal points, the front and rear principle points, and the front and rear nodal points, $N$ and $N^{\prime}$. For the purpose of modeling ocular parallax, we only require the front nodal point $N$, which is the center of projection of the eye, as well as the center of rotation $C$ (see Figure 2). Several schematic eye models have been proposed in the literature, each listing slightly different values for the cardinal points [Atchison 2017]. Some of the most popular models include the Gullstrand number 1, the Gullstrand-Emsley, and the Emsley reduced eyes, which are models of decreasing complexity. We outline the location of their respective nodal points and centers of rotation in Table 1. The center of rotation of the eye was measured to be $14.7536 \mathrm{~mm}$ from the cornea, on average, for emmetropic subjects [Fry and Hill 1962].
Table 1. Overview of Parameters of Popular Schematic Eye Models: Gullstrand Number 1 (Gull. 1), Gullstrand-Emsley (Gull.-Ems.), and Emsley Reduced (Ems.)

\begin{tabular}{|c|c|c|c|c|c|}
\hline & $\begin{array}{c}\text { Gull. 1 } \\
\text { Relaxed }\end{array}$ & $\begin{array}{c}\text { Gull. 1 } \\
\text { Acc. }\end{array}$ & $\begin{array}{c}\text { Gull.-Ems. } \\
\text { Relaxed }\end{array}$ & $\begin{array}{c}\text { Gull.-Ems. } \\
\text { Acc. }\end{array}$ & Ems. \\
\hline VN & 7.078 & 6.533 & 7.062 & 6.562 & 5.556 \\
\hline VN' $^{\prime}$ & 7.331 & 6.847 & 7.363 & 6.909 & 5.556 \\
\hline
\end{tabular}

The distances of front and rear nodal points $N, N^{\prime}$ are listed in mm with respect to the anterior vertex of the cornea $V$ for the relaxed and accommodated (acc.) state.

Although the specific locations of the cardinal points are slightly different for each eye model, the distance between center of rotation $C$ and center of projection $N$ is $7-8 \mathrm{~mm}$ in all cases. Note that nodal points for the Gullstrand number 1 and Gullstrand-Emsley models are accommodation dependent, i.e., the nodal points move slightly toward the cornea when the eye accommodates to close distances. However, in most current-generation VR/AR systems, the focal plane of the display is fixed. For example, the focal planes of the Oculus Rift and Microsoft Hololens are approximately $1.3 \mathrm{~m}$ and $2 \mathrm{~m}^{1}$ in front of the user, respectively. Since users will accommodate at that fixed focal distance [Padmanaban et al. 2017], we use the relaxed setting of the popular Gullstrand-Emsley eye model for all experiments in this article, i.e., $N C=7.6916 \mathrm{~mm}$.

Parallax and Acuity. We can model the amount of perceived ocular parallax expected in various viewing conditions. Similar to the illustration in Figure 1, we simulate two points that are directly in front of the eye at some relative distance to one another. As the eye rotates, the retinal images of these points will be perceived at an increasing eccentricity, or distance from the fovea, measured in degrees of visual angle. The larger the eccentricity, the larger the parallax, or relative distance, of the points on the retina. However, the density of photoreceptors, in particular the cones, decreases rapidly with increasing eccentricity. Thus, while one would expect a larger amount of parallax in the periphery, the threshold to perceive it there is higher due to the falloff in visual acuity.

Figure 3 illustrates the tradeoff between ocular parallax and visual acuity. Here, the minimum angle of resolution (MAR) represents a measure for visual acuity and approximates the resolution of human vision for a static target. To model falloff of acuity in the peripheral visual field, we use the linear model proposed by Guenter et al. [2012]: $\omega=m e+\omega_{0}$. Here, $\omega$ is the minimum angle of resolution (dashed red lines in Figure 3), $e$ is the eccentricity in degrees, $m$ is the slope modeling the falloff of acuity, and $\omega_{0}$ is the MAR at the fovea. We set $\omega_{0}=1 / 60$ to model $20 / 20$ vision and $m=0.022$ as proposed by Guenter et al. [2012]. As seen in Figure 3, a relative distance of $3 \mathrm{D}$ (diopters or inverse meters) should theoretically be detectable by a human observer for eccentricity angles smaller or equal to $40^{\circ}$.

The left part of Figure 3 also shows a semi-logarithmic plot zooming into the foveal region. We see that the magnitude of ocular parallax expected in the foveola, i.e., $e<1^{\circ}$, may not be sufficient to be perceivable. Yet for eccentricities larger than $1^{\circ}$, relative object distances of 2-3 D may make ocular parallax a useful depth cue. The dashed blue lines in Figure 3 also show the resolution of

${ }^{1}$ https://docs.microsoft.com/en-us/windows/mixed-reality/comfort. 


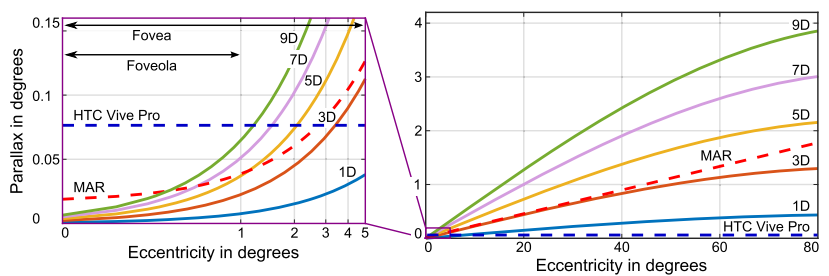

Fig. 3. The amount of ocular parallax, measured in degrees of visual angle increases with increasing eccentricity and relative distance between objects. Relative object distances of 3 diopters (inverse meters) and greater are above the minimum angle of resolution (MAR, red dashed line) and may therefore be detectable by a human observer. However, the amount of parallax on the foveola may be too small to be detected for static stimuli (left). The resolution available by modern VR displays, like the HTC Vive Pro (dashed blue lines), is slightly lower than the MAR of human vision in the foveola, but it exceeds the MAR for $2-3^{\circ}$ of visual angle-precisely where we expect to see the effects of ocular parallax.

the HTC Vive Pro, one of the highest-resolution VR displays available today. The size of one pixel of this display is approximately 4.58 arcmin of visual angle, which is about $5 \times$ higher than the MAR in the foveola. The resolution of this display starts to exceed the MAR at a distance of $2-3^{\circ}$ of visual angle, implying that this technology is well suited to render ocular parallax precisely where it is expected to be perceived by the user.

However, ocular parallax is a motion cue, and visual acuity alone may be insufficient to fully describe its perceived effects because that model is only valid for static scenes. During eye movement, the perceived depth-dependent motion created by parallax results in time-varying retinal stimuli. Detection thresholds for differential velocities of this type have been shown to be considerably lower than the limits of visual acuity for all retinal loci. For example, Mckee and Nakayama [1984] measured resolution thresholds of 2.7 arcmin and 4.8 arcmin in two participants at $10^{\circ}$ eccentricity, but found that their comparable motion thresholds were less than 1 arcmin, indicating that the visual acuity-based analysis above is an overly conservative estimate for the conditions in which ocular parallax may be detectable.

Retinal Blur. As discussed above, larger relative distances between objects result in an increasing amount of ocular parallax. For vision in the physical world, such increasing distances also result in an increasing amount of defocus blur because objects at different optical distances require the eye to accommodate at one of them, placing the other out of focus. However, current VR/AR displays provide a substantially different viewing condition in that they usually provide only a single focal plane at a fixed optical distance. Users must accommodate to this fixed distance to perceive a sharp image [Padmanaban et al. 2017]. With AR/VR displays, we have full control over how much blur to render into the presented images and may choose to ignore this rendering altogether as it adds substantial computational cost. While depth-offield rendering can be used to reduce visual discomfort in conventional stereo displays [Duchowski et al. 2014], it has not proven successful in driving accommodation or mitigating the vergenceaccommodation conflict [Johnson et al. 2016; Konrad et al. 2015; Mauderer et al. 2014], or acting as a reliable depth cue for single plane displays [Marshall et al. 1996; Mather and Smith 2002;
Palmer and Brooks 2008; Zannoli et al. 2016]. Without depth-offield rendering, ocular parallax is not affected by defocus blur in $\mathrm{VR} / \mathrm{AR}$, but it is still affected by peripheral falloffs in acuity and differential motion thresholds. In the following section, we model and render ocular parallax without retinal blur as a computationally efficient approximation.

\section{RENDERING OCULAR PARALLAX}

\subsection{Gaze-contingent Rendering}

In this section, we describe necessary modifications of the graphics pipeline to render ocular parallax.

Nodal Points. Ocular parallax is a gaze-contingent effect, and as such, eye tracking is necessary to render it appropriately. We assume that a binocular eye tracker estimates the 3D fixation point $\mathrm{F}$, which is typically defined with respect to the midpoint between the two eyes (see Figure 4). The center of rotation of each eye is offset from this midpoint by half the interpupillary distance (ipd). Defining the nodal points of each eye $\mathrm{N}_{L / R}$ relative to their respective center of rotation, they can be computed as

$$
\mathrm{F}_{L / R}=\mathrm{F} \pm\left(\begin{array}{c}
\frac{i p d}{2} \\
0 \\
0
\end{array}\right), \quad \mathrm{N}_{L / R}=\frac{N C}{\left|\mathrm{~F}_{L / R}\right|} \mathrm{F}_{L / R}
$$

where $\mathbf{F}_{L / R}$ defines the fixation point relative to each eye's center of rotation $\mathrm{C}$, and $\mathrm{NC}$ is the distance between the center of rotation and the front nodal point. The locations of these nodal points are then used to update the view and projection transforms in each rendered frame.

View and Eye Matrix. The standard graphics pipeline transforms each vertex $\mathbf{v}$ to view space by multiplying it with the model (M) and view (V) matrices. In binocular displays, such as VR and AR systems, an additional per-eye translation by half the ipd is applied to create correct stereoscopic cues by transforming vertices into eye space. To account for ocular parallax, we modify the transform to eye space with an additional translation by $-\mathrm{N}_{L / R}$. The full transformation from each vertex to eye space is then defined as

$$
\begin{gathered}
\mathbf{v}_{L / R}^{(\text {eye })}=\mathrm{E}_{L / R} \cdot \mathbf{V} \cdot \mathbf{M} \cdot \mathbf{v}, \\
\mathbf{E}_{L / R}=\left[\begin{array}{cccc}
1 & 0 & 0 & -N_{L / R}^{(x)} \\
0 & 1 & 0 & -N_{L / R}^{(y)} \\
0 & 0 & 1 & -N_{L / R}^{(z)} \\
0 & 0 & 0 & 1
\end{array}\right]\left[\begin{array}{cccc}
1 & 0 & 0 & \pm \frac{i p d}{2} \\
0 & 1 & 0 & 0 \\
0 & 0 & 1 & 0 \\
0 & 0 & 0 & 1
\end{array}\right],
\end{gathered}
$$

where $\mathrm{E}_{L / R}$ is the eye matrix, i.e., the transformation from view space to eye space, and $\mathbf{v}_{L / R}^{(e y e)}$ defines each vertex in eye space.

Projection Matrix. Vertices in eye space are transformed into clip space using the projection matrix. A perspective projection in stereo rendering is usually represented as an asymmetric off-axis view frustum defined by a near and far clipping plane, $z_{\text {near }}$ and $z_{f a r}$, as well as the left $(l)$, right $(r)$, top $(t)$, and bottom $(b)$ boundary values on the near clipping plane [Shirley et al. 2009]. Using a right-handed coordinate system, the corresponding projection 


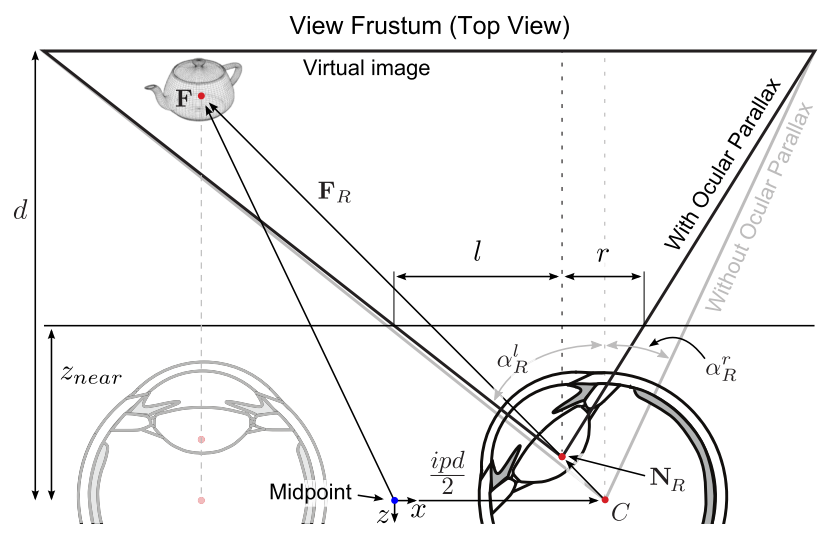

Fig. 4. Illustration of parameters used to compute the nodal points $\mathrm{N}$ for each eye, defined with respect to the center of rotation $\mathrm{C}$ of the respective eye, from the fixation point $\mathbf{F}$, which is estimated by the eye tracking. The precise locations of these nodal points are required for calculating the view and projection matrices in the rendering pipeline.

matrix has the general structure outlined in Equation (5). For clarity, we only show the projection transform of the right eye, but a matrix of similar form is applied for the left eye:

$$
\begin{gathered}
\mathbf{v}_{L / R}^{(\text {clip })}=\mathbf{P}_{L / R} \cdot \mathbf{v}_{L / R}^{(\text {eye }),} \\
\mathbf{P}_{R}=\left[\begin{array}{cccc}
\frac{2 \cdot z_{\text {near }}}{r_{R}-l_{R}} & 0 & \frac{r_{R}+l_{R}}{r_{R}-l_{R}} & 0 \\
0 & \frac{2 \cdot z_{\text {near }}}{t_{R}-b_{R}} & \frac{t_{R}+b_{R}}{t_{R}-b_{R}} & 0 \\
0 & 0 & \frac{-\left(z_{\text {far }}+z_{\text {near }}\right)}{z_{\text {far }}-z_{\text {near }}} & \frac{-2 \cdot z_{\text {far }} \cdot z_{\text {near }}}{z_{\text {far }}-z_{\text {near }}} \\
0 & 0 & -1 & 0
\end{array}\right] .
\end{gathered}
$$

The frustum boundary values are determined from parameters of the physical setup, including the field of view of the display, the distance to the virtual image $\mathrm{e}^{2} d$, and the position of the front nodal points $\mathrm{N}_{L / R}$ defined with respect to the corresponding center of rotation C. Assuming that we know the fields of view $\alpha^{\{l, r, t, b\}}$ defining the asymmetric frustum of the conventional stereo rendering mode (see Figure 4), we can compute the asymmetric view frustum of ocular parallax rendering as

$$
\begin{aligned}
& \left\{l_{R}, r_{R}\right\}=\frac{z_{\text {near }}+N_{R}^{(z)}}{d+N_{R}^{(z)}}\left(d \cdot \tan \left(\alpha_{R}^{\{l, r\}}\right)+N_{R}^{(x)}\right), \\
& \left\{t_{R}, b_{R}\right\}=\frac{z_{\text {near }}+N_{R}^{(z)}}{d+N_{R}^{(z)}}\left(d \cdot \tan \left(\alpha_{R}^{\{t, b\}}\right)+N_{R}^{(y)}\right) .
\end{aligned}
$$

The projection matrix is updated on a per-frame basis using the tracked nodal points. Applying the above modifications to the view and projection transforms renders perceptually accurate ocular parallax using slight modifications of the graphics pipeline, under the assumption that there are no optical distortions. Currently, we use the manufacturer-supplied optical distortion correction to account for optical aberrations of the HMD lenses across the visual field.

\footnotetext{
${ }^{2}$ We make the assumption that the virtual image is at optical infinity, i.e., $d=\infty$, such that distant objects do not move during eye motion but objects at closer distances shift relative to the background.
}

\subsection{Perceptual Effects of Ocular Parallax Rendering}

Ocular parallax rendering is expected to have implications on several depth cues. We briefly discuss these here to motivate the user experiments that specifically evaluate each of these effects in the following sections.

Micro Parallax. Parallax describes both magnitude and direction of the retinal velocity of objects at different depths while either the scene is in motion or the user's head moves. We expect a similar effect to occur, albeit at a significantly smaller magnitude, due to changes in gaze direction when ocular parallax rendering is enabled. Such changes in gaze direction rotate the nodal point about the center of rotation, inducing tiny amounts of depthdependent "micro parallax" into the retinal image. The relative magnitudes of the velocities of objects at different depths could provide an ordinal depth cue, helping users better understand the relative depth ordering of a scene. This has, for example, been shown to be the case for head motion parallax [Yonas et al. 1987], but it is not clear whether ocular parallax is an effective ordinal depth cue as well. Furthermore, the absolute magnitude of retinal velocity induced by ocular parallax could serve as an absolute depth cue, but the small retinal velocity magnitudes may not be sufficient to robustly estimate absolute distance. While micro parallax has an effect on all conducted user experiments, we specifically quantify its perceptual effect size in the discrimination threshold experiment in Section 5.2.

Gaze-contingent Occlusion. Micro parallax near occlusion boundaries is particularly interesting because there we observe gaze-contingent occlusion (see Figure 1). When objects at different depths overlap, the observed parallax due to eye rotations causes the accretion-deletion of only the farther object's texture. While occlusion can only provide ordinal depth information, it is considered one of the strongest depth cues in static environments [Cutting and Vishton 1995]. Particularly relevant to gazecontingent occlusion is the fact that time-varying accretiondeletion of texture due to head-motion-induced parallax has been shown to be an effective ordinal depth cue [Yonas et al. 1987]. Yet, it is unknown whether the same is true for the small amounts of accretion-deletion of texture observed with ocular parallax rendering. We evaluate the perceptual effect size of gaze-induced occlusion by estimating the detection thresholds for ocular parallax in Section 5.1 and its effectiveness as an ordinal depth cue in Section 6.1.

Disparity Distortion. Conventional stereoscopic rendering techniques assume that the centers of projection and rotation of the eyes are equivalent. We show that this is not the case. Therefore, ocular parallax could, in principle, also affect the rendered disparity values in stereographic image pairs and therefore distort perceived depth. We evaluate this hypothesis with a user experiment that studies the effect of ocular parallax on absolute depth perception in Section 6.2.

\subsection{Implementation}

Hardware. We implement ocular parallax rendering with a prototype VR system. The VR system is an HTC Vive Pro connected to the open source binocular Pupil Labs eye tracker. The HTC 
Vive Pro has a field of view of $110^{\circ}$, a refresh rate of $90 \mathrm{~Hz}$, and a $1,440 \times 1,600$ pixel organic light-emitting diode display, resulting in a theoretical resolution of $4.58 \mathrm{arcmin} /$ pixel. The HTC Vive Pro supports built-in ipd adjustment. The Pupil Labs eye tracker snaps into the the HTC Vive Pro and estimates a global fixation point (i.e., F) at $120 \mathrm{~Hz}$, supporting about $1^{\circ}$ of manufacturer-reported gaze accuracy and $0.08^{\circ}$ of gaze precision (see supplement for additional characterization). In the egocentric depth perception study, an HTC Vive Tracker estimates the position and orientation of the users' hand at $120 \mathrm{~Hz}$ with an accuracy of $1.9 \mathrm{~mm}$ root-meansquare-error (RMSE) and a precision of $1.5 \mathrm{~mm} \mathrm{RMSE}{ }^{3}$

Software and Calibration. Unity was used as the rendering engine for both the ocular parallax rendering and the user experiments. Pixel-precise rendering and anti-aliasing were used for all rendered stimuli. Pupil Labs provides a Unity plugin that interfaces with their Python library, providing eye-tracking calibration and gaze tracking. All software related to acquiring subject data was written as C\# scripts in Unity. The data were then analyzed in Python.

\section{PERCEPTUAL THRESHOLDS FOR OCULAR PARALLAX IN VR}

The primary goal of this section is to establish depth-dependent detection and discrimination thresholds for ocular parallax in VR. Although ocular parallax has been shown to be well within the range of human visual acuity for natural viewing conditions (e.g., Bingham [1993]), we are not aware of any work that actually verified that this small effect size is even perceivable with the limited resolution offered by current-generation VR displays or, if it is, what the specific thresholds are. These thresholds are crucial for understanding in which conditions ocular parallax rendering is visible and how reliable of a depth cue it may be. To estimate these thresholds, we perform two psychophysical experiments that are discussed in the following sections with the apparatus described in Section 4.3.

\subsection{Detection Thresholds for Ocular Parallax}

With this experiment, we aim to estimate a depth-dependent detection threshold at which ocular parallax is perceivable.

Stimuli. As seen in Figure 5 (left), we presented two circular surfaces at varying distances to the subject. The surfaces were scaled to subtend $2^{\circ}$ of visual angle irrespective of depth. The farther surface was solid red and the front one was textured with white noise. Without ocular parallax enabled, the front surface exactly occluded the back one.

An additional small red and white fixation target was then rendered, circling around the scene at a distance of $16^{\circ}$ from the center, or $15^{\circ}$ from the edges of the front and back surfaces. This was the largest eccentricity that kept the target within the aberration-free viewing zone of the Vive Pro. This fixation target rotated with an angular velocity of $90 \circ / \mathrm{s}$, resulting in a perceived retinal motion of $24.81 \circ / \mathrm{s}$, which falls within smooth pursuit rates [Westheimer 1954]. The starting position and rotation direction of the fixation target were randomized per trial.

\footnotetext{
${ }^{3}$ http://doc-ok.org/?p=1478.
}

Conditions. All stimuli were presented monocularly to the right eye. In each trial, the absolute distance between the viewer and back surface was randomly chosen as 1,2 , or $3 \mathrm{D}$. The relative distance between the front and back surface was also randomly chosen as $0,0.25,0.5,0.75$, or $1 \mathrm{D}$. In each trial, subjects viewed the stimulus with ocular parallax rendering enabled and disabled. These two conditions were presented in different 2-second intervals in random order, separated by a 0.5 second blank frame.

Subjects. Six adults participated (age range 26-38, 1 female). Due to the demanding nature of our psychophysical experiment, only a few subjects were recruited, which is common for low-level psychophysics (see, e.g., Patney et al. [2016]).

All subjects in this and all following experiments had normal or corrected to normal vision, no history of visual deficiency, and no color blindness. All subjects gave informed consent. The research protocol was approved by the Institutional Review Board at the participating university.

Procedure. To start the session, each subject performed a 7-point eye-tracker calibration that is provided by the manufacturer. To minimize eye-tracker error over the course of the experiment, each trial began with a single-point re-calibration of the eye trackers; subjects were instructed to fixate on a cross target centered on the screen, and the measured gaze direction was used to compensate for possible drift error. Subjects then viewed the stimulus rendered with one of the two ocular parallax rendering conditions for 2 seconds, then a blank screen for 0.5 seconds, and then again the stimulus with the other rendering condition from the first interval for another 2 seconds. Each trial constituted a twoalternative forced choice test, and subjects were asked to choose the time interval that exhibited more relative motion between the two surfaces with a keyboard. This concluded the trial. No feedback was provided. Subjects were instructed to fixate only on the moving fixation target, and never to the center surfaces.

There were 15 distance configurations and 15 trials for each configuration for a total of 225 trials per subject. The experiment took about 25 minutes to complete, including instruction and eye tracking calibration per subject.

Analysis. For each of the 15 distance configurations, we computed the proportion of correct responses. Using Bayesian inference methods [Schütt et al. 2016; Wichmann and Hill 2001a, $2001 \mathrm{~b}$ ], we fit a psychometric function to each subject's performance at each of the three absolute depths of the back surface. Each psychometric function gives us a detection threshold, measured in diopters of relative distance from the absolute distance of the back surface. The thresholds represent where the psychometric function exceeded a 75\% chance for a correct response. An example of one of these psychometric functions is shown in Figure 5 (top right), and all measured psychometric functions are available in the supplement.

Results. The detection thresholds, averaged across subjects, are plotted in Figure 5 (center left). We see that these thresholds are invariant to the absolute distance of the back surface. This is expected because the conditions were equally spaced in diopters, and ocular parallax, like other types of parallax cues, is perceptually linear in dioptric space (see supplement for an analysis). The 

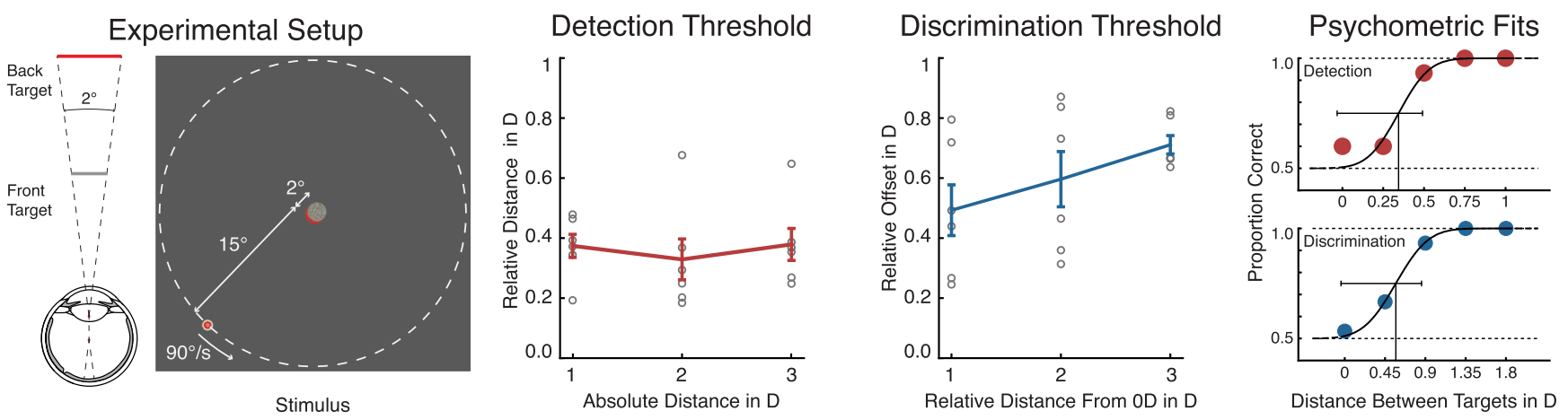

Fig. 5. Detection and discrimination thresholds for ocular parallax in VR. Two experiments were conducted to estimate perceptual thresholds using an HTC Vive Pro head-mounted display presenting stimuli of the form shown on the left. A red surface subtending $2^{\circ}$ of visual angle was completely occluded by a noisy gray surface in front of it (left). The relative distances of these surfaces were varied with conditions described in the text. Detection thresholds (center left) and discrimination thresholds (center right) were estimated from the psychometric functions fitted to the recorded subject data (examples shown right).

estimated detection threshold is approximately $0.36 \mathrm{D}$. This implies that, for an eccentricity of $15^{\circ}$ and a relative object distance as low as $0.36 \mathrm{D}$, ocular parallax may be perceivable in VR.

This result is surprising because visual acuity alone (see Figure 3) predicts detection thresholds that are an order of magnitude higher that what we measured. Yet, our results are consistent with data reported for physical viewing conditions (i.e., non-VR settings) [Bingham 1993] and with differential velocity thresholds [Mckee and Nakayama 1984]. We expect the detection thresholds to increase linearly with eccentricity in the extrafoveal region because both the magnitude of micro parallax (see Figure 3) and differential velocity thresholds increase roughly linearly there [Mckee and Nakayama 1984]. Also, we expect detection thresholds to decrease with faster eye movements because differential velocity thresholds inversely vary with retinal velocity [Mckee and Nakayama 1984].

Our results emphasize that the human visual system is much more sensitive to small amounts of motion, even in peripheral vision, than naïvely expected. Even the small amount of parallax induced by ocular parallax rendering may be visible in many VR applications. An important question that arises from this insight is whether ocular parallax rendering can improve depth perception or the realism of a 3D scene. We perform several experiments in Sections 6 and 7 that aim at answering this question.

\subsection{Discrimination Thresholds for Ocular Parallax}

A discrimination threshold tells us what the smallest amount of perceivable change in ocular parallax is. For a fixed eye eccentricity, this threshold depends on both the absolute distance of the reference surface (i.e., the back surface) and also the relative offset from that surface. Conceptually, one would have to estimate discrimination thresholds for each combination of absolute and relative distance, which would be an arduous task. However, due to the fact that the detection thresholds are depth independent, we assume that the discrimination thresholds are also independent of the absolute distance to the back surface. This assumption makes it easier to set up an experiment to estimate discrimination thresholds for ocular parallax in VR, which we did by performing a second experiment that uses the same apparatus, stimuli, and analysis as the first experiment and a very similar procedure, but with slightly different conditions. Six adults participated (age range 26-32, 1 female).

Conditions. The back surface was fixed to $0 \mathrm{D}$ for all conditions. Instead of presenting the stimulus with ocular parallax rendering enabled for only one interval, as done in the previous experiment, we enabled ocular parallax rendering for both intervals. The relative offset from the back surface was randomly chosen as 1,2 , or $3 \mathrm{D}$ and assigned as the depth for one of the front surfaces shown in each trial. The other appeared at one of the following distances from the previously chosen relative offset: $0,0.45,0.9,1.35$, and $1.8 \mathrm{D}$ for the $1 \mathrm{D}$ and $2 \mathrm{D}$ initial offsets; and $0,0.7,1.4,2.1$, and $2.8 \mathrm{D}$ for the $3 \mathrm{D}$ initial offset. Again, there were 15 distance configurations and 15 trials for each configuration for a total of 225 trials per subject.

Results. We used a similar analysis as the one described in the previous section to estimate the discrimination thresholds, which are plotted in Figure 5 (center right). As expected, the discrimination thresholds increase linearly with increasing amounts of ocular parallax, per Weber's law, but due to the proximity to the detection threshold magnitude, the slope is less than 1. A linear fit between the discrimination thresholds and relative offset from $0 \mathrm{D}$ has a slope of 0.11 and an intercept of $0.38 \mathrm{D}$, further verifying the measured $0.36 \mathrm{D}$ detection threshold from the previous experiment.

In conclusion, the estimated discrimination thresholds are well within the range of the depth ranges of natural scenes commonly rendered for immersive VR applications. This result motivates further studies to investigate if or when ocular parallax could be used as a reliable depth cue in these applications. We take a first step at answering this question by conducting experiments that study whether ocular parallax has a measurable effect on depth perception in the following sections.

\section{OCULAR PARALLAX AND DEPTH PERCEPTION}

Motivated by the discussion in Section 4.2 and the surprisingly low detection and discrimination thresholds measured in the previous section, we proceed to investigate the importance of ocular parallax as a depth cue. Here, we distinguish between ordinal depth 
perception, which provides information about the relative depth ordering of a scene (i.e., object A is closer than object B), and metrical depth cues, which also provide absolute distance estimates (i.e., object $\mathrm{A}$ is $1 \mathrm{~m}$ away and object $\mathrm{B}$ is $2 \mathrm{~m}$ away). It seems intuitive that gaze-contingent occlusion can provide ordinal depth information due to the deletion and accretion of the farther object's texture near occlusion boundaries. Moreover, gaze-induced micro parallax also provides ordinal information in the relative magnitudes of retinal velocities of objects at different depths. However, the effectiveness of ocular parallax as an absolute depth cue is questionable because detecting absolute motion velocities of objects at different depths may be unreliable, and occlusion only provides ordinal depth information. We investigate the effect of ocular parallax rendering on both ordinal and absolute depth perception in the following experiments with the apparatus described in Section 4.3.

\subsection{Effect of Ocular Parallax on Ordinal Depth Estimation}

In this section, we study the benefits of ocular parallax rendering on ordinal depth estimation. We also investigate whether depth perception gained from ocular parallax is purely a visual process, or requires some non-visual, extra-retinal signal like the magnitude or direction of eye rotation. The perception of depth from head-motion parallax, for example, has been shown to rely on an extra-retinal signal in the form of optokinetic response eye movements [Nawrot 2003]. This experiment is modeled after a related experiment investigating retinal blur as an ordinal depth cue [Zannoli et al. 2016]. Twenty-one adults participated (age range 22-43, 4 females), of which two were excluded for failing to follow instructions.

Stimuli. The monocular stimuli, displayed on a single virtual image plane, consisted of two differently textured, frontoparallel surfaces at different depths (Figure 6, top). The textures, the same as those in the aforementioned depth order study [Zannoli et al. 2016], had the same space-average luminance and contrast energy, and exhibited similar amplitude spectra. The rear surface was fixed at $0.5 \mathrm{D}$ and the front surface appeared at either $1.5 \mathrm{D}$ or $2.5 \mathrm{D}$. The border between the two surfaces had a sinusoidal shape. The surfaces were scaled to subtend the same visual angle irrespective of depth. Subjects viewed the surfaces through a $20^{\circ}$ circular aperture that was unaffected by ocular parallax rendering.

Procedure. First, all subjects performed a 7-point eye-tracker calibration provided by the manufacturer to start the session. As in the psychophysical experiments, each individual trial began with a single-point re-calibration of the eye trackers. Following the recalibration, the stimulus was presented monocularly to the right eye for 3 seconds with one of the three rendering conditions. Subjects were instructed to freely gaze anywhere within the aperture where both surfaces were visible. The stimulus was then replaced with a prompt asking which surface, left or right, appeared nearer, and a keyboard response concluded the trial. No feedback was provided.

Conditions. We evaluated three different rendering conditions (conventional, ocular parallax, and reversed ocular parallax) and two near surface distances $(1.5 \mathrm{D}$ and $2.5 \mathrm{D})$. With the monocular

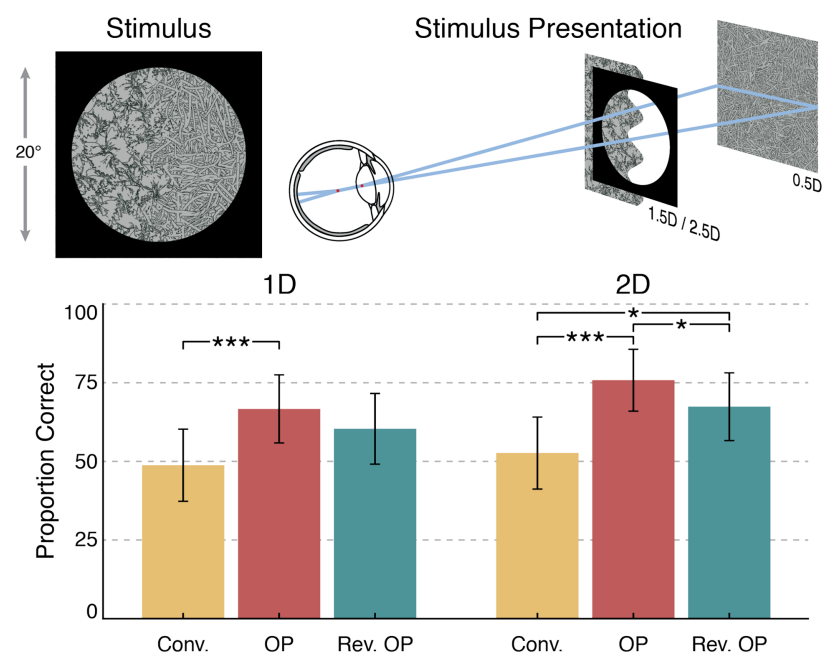

Fig. 6. Effect of ocular parallax on ordinal depth perception. Subjects viewed the monocular stimuli consisting of two distinctly textured surfaces separated by 1 D or 2 D (top) and were asked which one was closer. The proportions of correct responses, averaged across subjects per condition, are plotted on the bottom. Subjects performed significantly better with ocular parallax rendering enabled compared to conventional rendering. However, they also performed slightly better than conventional rendering with reversed ocular parallax rendering, indicating that the extraretinal signal of eye rotation may not be crucial for depth perception. Significance is indicated at the $p \leq 0.05,0.01$, and 0.001 levels with $*, * *$, and $* * *$, respectively. Error bars represent standard error.

stimuli devoid of perspective and accommodation/retinal blur cues, the only expected sources of depth information are gazecontingent micro parallax and occlusion. We included both correct and reversed ocular parallax rendering to understand whether depth perception in this scenario is purely a visual process, requiring only the retinal image with micro parallax, or whether it also requires an extra-retinal signal in the form of eye rotation direction. With reversed ocular parallax rendering, the sign of the visual signal is negated, causing occluded surface textures to accrete when they would normally be deleted. Subjects expecting the visual and extra-retinal signals to be consistent could misinterpret depth orderings. Reversed ocular parallax was implemented by negating the $x$ and $y$ components of the estimated nodal points of the eyes, $\mathrm{N}_{L / R}$.

The near surface distances create a $1 \mathrm{D}$ and $2 \mathrm{D}$ separation from the rear surface, resulting in different magnitudes of the gazecontingent effects. Following the threshold experiments, we expect the proportion of correct responses to increase with surface separation. Overall, there were 6 conditions and each condition was evaluated with 15 trials for a total of 90 trials per subject.

Results. The proportion of correct responses, averaged across subjects per condition, is plotted in Figure 6 (bottom). As expected, subjects in the conventional static rendering condition performed close to random, correctly identifying the nearer surface in $48.8 \%$ and $52.6 \%$ of trials for $1 \mathrm{D}$ and $2 \mathrm{D}$ of surface separation, respectively. Enabling ocular parallax rendering clearly improved ordinal depth judgment with subjects performing at $66.7 \%$ and $75.8 \%$ correct identification for the $1 \mathrm{D}$ and $2 \mathrm{D}$ separations, respectively. 
Subjects in the reversed ocular parallax condition fell in-between, performing at $60.4 \%$ and $67.4 \%$ correct identification for the two separation distances.

We conducted a $2 \times 3$ repeated-measures ANOVA on the proportion of correct responses with independent variables of rendering mode (conventional, ocular parallax, reversed ocular parallax) and separation distance (1 D or $2 \mathrm{D})$. Greenhouse-Geisser sphericity correction was applied. The ANOVA shows a very significant effect of rendering mode $(F(1.7,30.65)=17.98, p<0.0001)$ as well as a significant effect of distance $(F(1,18)=8.14, p<0.05)$. The ANOVA does not reveal a significant interaction between rendering mode and distance $(F(1.78,31.96)=0.42, p=0.64)$.

Post-hoc tests were conducted as pairwise $t$-tests between rendering modes at each separation distance, with Bonferroni correction applied to the $p$-values. The post-hoc tests found that, at $1 \mathrm{D}$ of separation, ocular parallax rendering shows a significant improvement over conventional rendering $(p<0.001)$, but not over reversed ocular parallax rendering. Reversed ocular parallax rendering does not show a significant improvement over conventional rendering. At $2 \mathrm{D}$ of separation, ocular parallax rendering shows a significant improvement over conventional rendering $(p<0.001)$ as well as reversed ocular parallax rendering $(p<0.05)$. Reversed ocular parallax rendering also shows a significant improvement over conventional rendering $(p<0.05)$ at this separation.

In summary, this experiment demonstrates that ocular parallax significantly improves ordinal depth perception over conventional rendering. However, reversed ocular parallax also improves ordinal depth perception over conventional rendering, but not nearly as much as when rendered correctly. The reduced performance in the reversed ocular parallax condition compared to the correct ocular parallax condition suggests that extra-retinal signals, like eye rotation, play an important role in the perception of depth. However, this effect seems to be weaker than for motion parallax, where a reversal in eye movement causes a reversal in the sign of the perceived depth [Nawrot 2003]. Still, subjects performed better, in some conditions significantly so, when the directions of the retinal image motion and eye rotation were consistent. Therefore, enabling correct ocular parallax rendering can benefit ordinal depth estimation, which can be particularly useful for viewing 3D scenes that often contain many occlusion boundaries.

\subsection{Effect of Ocular Parallax on Absolute Depth Estimation}

Next, we study whether ocular parallax rendering can benefit egocentric distance (distance from one's self) estimation in a stereo environment. While unlikely, gaze-induced micro parallax and the reduction in peripheral disparity errors could affect absolute distance estimation.

In the experiment, subjects performed a blind reaching task introduced by Napieralski et al. [2011] to estimate distances to objects because verbal estimates have been shown to be inaccurate [Renner et al. 2013]. In a photo-realistic dining room scene, subjects viewed a stereoscopically rendered pencil for a minimum of 5 seconds after which the screen was blanked and they reached their hand to where they had last seen the pencil (Figure 7, top left). Each subject performed the task with ocular parallax enabled and disabled, and the reach target distances were
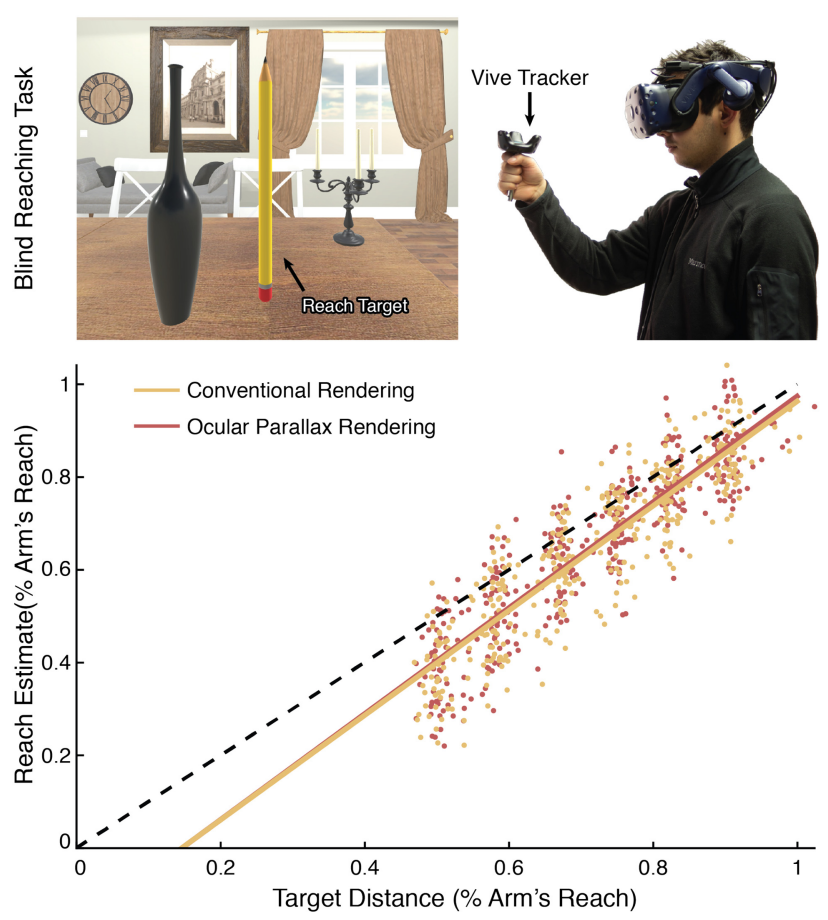

Fig. 7. Effect of ocular parallax on absolute, egocentric depth perception. Subjects viewed a target (pencil) and then, with the display turned off, were asked to reach for it (top right). We did not find a statistically significant difference between conventional stereo rendering and stereo rendering with ocular parallax in this experiment (bottom plot).

set proportionally-50,58, 67, 75, 82, and 90\%-to each subjects' maximum arm reach. Each of the 12 conditions were evaluated with five blind reaching tasks for a total of 60 randomly-ordered trials per subject. During the session, 6-degree-of-freedom head pose tracking was enabled, and the hand's position was tracked via an HTC Vive tracker mounted on an optical post (Figure 7, top right). Sixteen young adults participated (age range 22-32, 5 females), of which one was excluded for not passing a standard Randot stereo vision test, and two others were excluded due to the eye tracker failing to track their pupils.

Subjects saw little to no improvement in their egocentric distance estimates with ocular parallax rendering enabled. Figure 7 shows each subject's reach, as a percentage of their maximum arm reach, to a presented target distance for the ocular parallax enabled and disabled conditions. Linear models were fit to the two sets of reaches; the slopes for the ocular parallax enabled and disabled modes were 1.141 and 1.128 , respectively, while the intercepts were 0.167 and 0.165 , respectively. A multiple regression analysis did not show a significant difference between the two conditions. It is therefore unlikely that the systematic underestimation of distances to virtual objects compared to real ones [Renner et al. 2013] can be explained by the omission of ocular parallax rendering. For more details regarding this experiment, please refer to the supplement.

\section{OCULAR PARALLAX AND PERCEPTUAL REALISM}

We next measured the effect of ocular parallax on perceptual realism. Twenty-one adults participated in the experiment (age range 


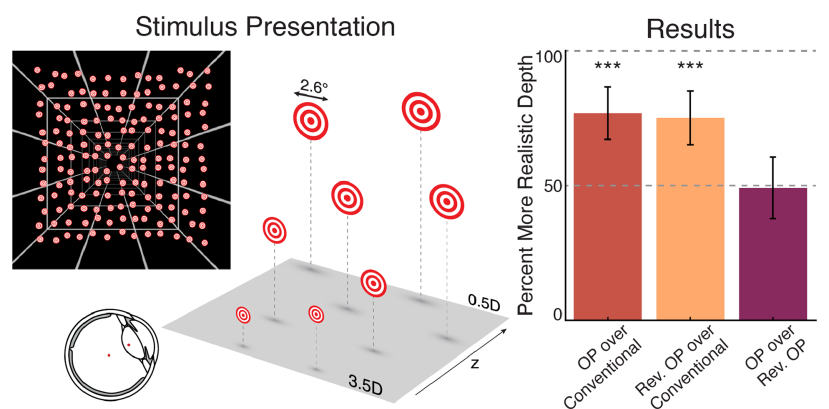

Fig. 8. Evaluating perceptual realism. Subjects viewed a 3D scene consisting of targets that are randomly distributed in depth but that do not occlude one another (left). This stimulus was presented with either conventional, ocular parallax, or reversed ocular parallax rendering, and we asked subjects to indicated which rendering mode provided a stronger impression of realistic depth. Results of pairwise comparisons between these rendering modes show the percent of times the first member of the pair was chosen over the second (right). Rendering with correct and reversed ocular parallax conveyed a stronger impression of depth compared to conventional rendering, but when compared against one another, no difference was observed. This result indicates that the relative magnitudes of depth-dependent motion velocities are most important for perceptual realism but not necessarily their direction. Significance is indicated at the $p \leq 0.05,0.01$, and 0.001 levels with $*, * *$, and $* * *$, respectively. Error bars represent standard error.

22-43, 4 females), of which two were excluded for failing to follow instructions.

Stimuli and Conditions. A 3D scene (Figure 8, left) was presented to the right eye with conventional, ocular parallax, or reversed ocular parallax rendering. The scene was composed of four grid planes extending into the distance and a field of frontoparallel targets. These targets were randomly distributed in depth between $0.5 \mathrm{D}$ and $3.5 \mathrm{D}$ and were scaled to subtend $2.6^{\circ}$ of visual angle regardless of depth. The targets' lateral positions were randomly chosen such that they did not occlude one another and the entire target field subtended $60^{\circ}$.

Procedure. First, all subjects performed a 7-point eye-tracker calibration provided by the manufacturer to start the session. As in the psychophysical experiments, each individual trial began with a single-point re-calibration of the eye trackers. Then, the target positions used for the subsequent stimuli were generated. The first stimulus was presented for 3 seconds with one of the three rendering conditions. The targets then disappeared for 1 second, leaving only the grid pattern shown with conventional rendering. The second stimulus was then presented for 3 seconds using one of the three rendering conditions, but not the same as the one used for the first stimulus. After the second stimulus, a prompt appeared asking the subjects to determine, in a forced-choice judgment, the stimulus that portrayed a stronger impression of realistic depth. No feedback was provided. Each trial therefore consisted of three pairwise comparisons: conventional vs. ocular parallax, conventional vs. reversed ocular parallax, and ocular parallax vs. reversed ocular parallax.

Results. The results of the pairwise comparisons are averaged across users and trials and plotted in Figure 8 (right). In $76.8 \%$ of trials, subjects reported a stronger sense of realistic depth when viewing ocular parallax rendering over conventional rendering. Interestingly, subjects also reported more realistic depth for reversed ocular parallax rendering over conventional rendering in $75.1 \%$ of trials. Each of these percentages is significantly greater than $50 \%$ ( $p<0.001$ and $p<0.001$, respectively, one-tailed binomial test). Users had more difficulty differentiating between the two ocular parallax modes and reported more realistic depth when ocular parallax was correctly rendered in only $49.1 \%$ of trials, which was not significantly greater than $50 \%$.

These results suggest that gaze-induced micro parallax, offered by ocular parallax rendering, has a significant effect on perceptual realism when compared to conventional rendering. Moreover, the results suggest that the relative motion magnitudes of the depth-dependent retinal velocities are more important than their direction. This insight further emphasizes that, similar to the ordinal depth perception task (Section 6.1), extra-retinal signals were likely not notably factored into the perception of depth on this subjective task. Unlike the experiment in Section 6.1, where both gazecontingent occlusion and micro parallax were present as cues, in this experiment, micro parallax was the primary indicator of depth because the targets did not overlap.

\section{DISCUSSION}

In summary, our primary contribution is to introduce a new technology for virtual and augmented reality: ocular parallax rendering. This technique is enabled by eye tracking systems that can already be found in some of the latest headsets, like the Microsoft Hololens 2, Magic Leap One, Varjo, Fove, and HTC Vive Pro Eye. Ocular parallax rendering could be jointly implemented with other gaze-contingent rendering methods, such as foveated rendering, and it requires no additional computational cost compared to conventional stereo rendering.

To evaluate ocular parallax rendering, we designed and conducted a series of user experiments. First, we measured detection thresholds and show that the effect is perceivable when the relative distance between two partially occluding objects is as low as 0.36 diopters. We also measured discrimination thresholds and confirm that the just noticeable difference between two objects at different relative depths in front of a background stimulus is directly proportional to their absolute depth from the background. These thresholds confirm that ocular parallax is an import visual cue in VR. Our third and fourth experiments show that ocular parallax acts as reliable ordinal depth cue but that it may not be a reliable absolute depth cue. Finally, our fifth experiment demonstrates that ocular parallax rendering significantly improves the impression of realistic depth when viewing a 3D scene over conventional rendering.

Ocular Parallax and Retinal Blur. Retinal blur describes the depth-dependent defocus blur of objects on the retina, relative to the accommodation distance. In physical environments, retinal blur discrimination thresholds are comparable to those of ocular parallax-approximately \pm 0.35 D [Ogle and Schwartz 1959]-and have been shown to serve as both a reliable ordinal and absolute depth cue [Vishwanath and Blaser 2010]. While recent research efforts in VR/AR have studied focus cues such as retinal blur, 
chromatic aberrations, and accommodation, none have studied ocular parallax, even though their effect sizes are very similar.

Gaze-contingent retinal blur and ocular parallax rendering can certainly be implemented simultaneously as they are complementary cues. While depth-of-field rendering blurs objects at different depths, we do not expect it to alter our measurements or insights significantly. This is because the perception of motion is understood to use low spatial frequencies [Smith and Snowden 1994], so the loss of the high spatial frequencies due to depth-of-field rendering should not impair motion, or ocular parallax, perception. Indeed, our measured detection thresholds are consistent with those measured in a physical environment [Bingham 1993], where retinal blur was apparent.

Eye Model. Throughout the article, we use a schematic eye that makes several simplifying assumptions. First, we assume that the front nodal point is indeed the center of projection. Second, we assume that the user does not accommodate. Third, we assume that the optical and visual axis of the eye are the same. The first assumption is reasonable and in line with the GullstrandEmsley schematic eye. However, it could be argued that there is no exact center of projection in the eye, that it varies significantly between users, or that it is the center of the entrance pupil of the eye, which laterally shifts with changing pupil diameter [Atchison and Mathur 2014], instead of the front nodal point. The precise location of the center of projection is a topic that deserves further discussion and that should also be experimentally located, which we leave for future work. The second assumption requires the user to accommodate at a fixed, far distance. For near-eye displays that support accommodation, vergence or accommodation tracking could be used to model the accommodation-dependent nodal point. Finally, we assume that optical and visual axis of the eye are the same. Using this assumption, we demonstrate that ocular parallax rendering has no significant effect on disparity distortion, thus absolute depth perception, in Section 6. However, further studies on this topic should be conducted using more accurate eye models that include, for example, an offset between visual and optical axis of the eye.

Limitations. Although our system uses some of the highestend components available, including a high-resolution wide-fieldof-view VR display and a $120 \mathrm{~Hz}$ eye tracker, the latency of $20 \mathrm{~ms}$ for gaze-contingent ocular parallax rendering is high. Faster and more accurate eye tracking would certainly help improve the user experience for all gaze-contingent rendering schemes, including ocular parallax.

Applications. We envision ocular parallax rendering to be a standard part of the graphics pipeline of eye-tracking-enabled near-eye displays. It improves perceptual realism, ordinal depth perception, and it may offer other perceptual benefits. In particular, optical see-through augmented reality systems may benefit from ocular parallax rendering as a user usually sees a digitally rendered stimulus overlaid on a reference stimulus (i.e., the physical world); visual cue consistency between these stimuli may be even more important than in VR. Finally, one could also imagine that an amplified version of ocular parallax rendering could be an effective gaze-contingent user interface that allows users to transform objects, navigate through virtual environments, or perform other tasks. Tasks that require hands-free operation could particularly benefit from this type of gaze-contingent interaction mode.

Future Work. As the fields of view of emerging VR and AR systems keep increasing, understanding perceptual effects in peripheral vision becomes ever more important. With this work, we thoroughly evaluate the perceptual implications of one technique, ocular parallax rendering, which shows the strongest effects in near-mid peripheral vision. However, many other technologies, such as foveated rendering, reducing motion sickness, or other means to improve depth perception or perceptual realism, could also benefit from studying their perceptual effects on peripheral vision. Additionally, as many of these effects vary from person to person, performing a per-user calibration of the distance between the centers of rotation and projection could further increase perceptual realism.

Conclusions. Virtual and augmented reality systems have focused on improving resolution, field of view, device form factor, and other characteristics. With this work, we hope to stimulate new directions for gaze-contingent rendering and improve perceptual realism and depth perception with next-generation near-eye displays.

\section{REFERENCES}

Kurt Akeley, Simon J. Watt, Ahna R. Girshick, and Martin S. Banks. 2004. A stereo display prototype with multiple focal distances. ACM Trans. Graph. (SIGGRAPH) 23, 3 (2004), 804-813.

David A. Atchison. 2017. Schematic eyes. In Handbook of Visual Optics, Volume IFundamentals and Eye Optics, Pablo Artal (Ed.). CRC Press, Chapter 16.

David A. Atchison and Ankit Mathur. 2014. Effects of pupil center shift on ocular aberrations. Invest. Ophthalmol. Visual Sci. 55, 9 (2014), 5862-5870.

Geoffrey P. Bingham. 1993. Optical flow from eye movement with head immobilized: "Ocular occlusion" beyond the nose. Vision Res. 33, 5 (1993), 777-789.

David Brewster. 1845. On the law of visible position in single and binocular vision, and on the representation of solid figures by the union of dissimilar plane pictures on the retina. In Proc. Royal Society of Edinburgh 1 (1845).

Steven A. Cholewiak, Gordon D. Love, Pratul P. Srinivasan, Ren Ng, and Martin S. Banks. 2017. Chromablur: Rendering chromatic eye aberration improves accommodation and realism. ACM Trans. Graph. (SIGGRAPH Asia) 36, 6 (2017), 210:1210:12.

James Cutting and Peter Vishton. 1995. Perceiving layout and knowing distances: The interaction, relative potency, and contextual use of different information about depth. In Perception of Space and Motion, William Epstein and Sheena Rogers (Eds.). Academic Press, Chapter 3, 69-117.

Piotr Didyk, Tobias Ritschel, Elmar Eisemann, Karol Myszkowski, and Hans-Peter Seidel. 2011. A perceptual model for disparity. ACM Trans. Graph. (SIGGRAPH) 30, 4 (2011), 96:1-96:10.

Andrew T. Duchowski, Nathan Cournia, and Hunter A. Murphy. 2004. Gazecontingent displays: A review. Cyberpsychol. Behav. 7 (2004), 621-34.

Andrew T. Duchowski, Donald H. House, Jordan Gestring, Rui I. Wang, Krzysztof Krejtz, Izabela Krejtz, Radoslaw Mantiuk, and Bartosz Bazyluk. 2014. Reducing visual discomfort of 3D stereoscopic displays with gaze-contingent depth-of-field. In ACM Symposium on Applied Perception. 39-46.

David Dunn, Cary Tippets, Kent Torell, Petr Kellnhofer, Kaan Aksit, Piotr Didyk, Karol Myszkowski, David Luebke, and Henry Fuchs. 2017. Wide field of view varifocal near-eye display using see-through deformable membrane mirrors. IEEE TVCG 23, 4 (2017), 1322-1331.

Glenn A. Fry and W. W. Hill. 1962. The center of rotation of the eye. Optometry Vision Sci. (1962), 581-595.

Brian Guenter, Mark Finch, Steven Drucker, Desney Tan, and John Snyder. 2012 Foveated 3D graphics. ACM Trans. Graph. (SIGGRAPH Asia) 31, 6 (2012), 164:1164:10.

Itzhak Hadani, Gideon Ishai, and Moshe Gur. 1980. Visual stability and space perception in monocular vision: Mathematical model. OSA f. Opt. Soc. Am. 70, 1 (1980), 60-65.

David M. Hoffman, Ahna R. Girshick, Kurt Akeley, and Martin S. Banks. 2008. Vergence-accommodation conflicts hinder visual performance and cause visual fatigue. 7. Vision 8, 3 (2008), 33.

Ian P. Howard and Brian J. Rogers. 2002. Seeing in Depth. Oxford University Press. 
Xinda Hu and Hong Hua. 2014. High-resolution optical see-through multi-focal-plane head-mounted display using freeform optics. Opt. Express 22, 11 (Jun 2014), 1389613903.

Hong Hua and Bahram Javidi. 2014. A 3D integral imaging optical see-through headmounted display. Optics Express 22, 11 (2014), 13484-13491.

Fu-Chung Huang, Kevin Chen, and Gordon Wetzstein. 2015. The light field stereoscope: Immersive computer graphics via factored near-eye light field display with focus cues. ACM Trans. Graph. (SIGGRAPH) 34, 4 (2015).

Paul V. Johnson, Jared A. O. Parnell, Joohwan Kim, Christopher D. Saunter, Gordon D. Love, and Martin S. Banks. 2016. Dynamic lens and monovision 3D displays to improve viewer comfort. OSA Opt. Express 24, 11 (2016), 11808-11827.

Petr Kellnhofer, Piotr Didyk, Tobias Ritschel, Belen Masia, Karol Myszkowski, and Hans-Peter Seidel. 2016. Motion parallax in stereo 3D: Model and applications. ACM Trans. Graph. (SIGGRAPH) 35, 6 (2016), 176:1-176:12.

Robert Konrad, Emily Cooper, and Gordon Wetzstein. 2015. Novel optical configurations for virtual reality: Evaluating user preference and performance with focustunable and monovision near-eye displays. In Proc. SIGCHI.

Hiroaki Kudo and Noboru Ohnishi. 1998. Study on the ocular parallax as a monocular depth cue induced by small eye movements during a gaze. In Proc. IEEE Engineering in Medicine and Biology Society, Vol. 6. 3180-3183.

Hiroaki Kudo and Noboru Ohnishi. 2000. Effect of the sight line shift when a headmounted display is used. In Proc. EMBS International Conference, Vol. 1. 548550.

Hiroaki Kudo, Masaya Saito, Tsuyoshi Yamamura, and Noboru Ohnishi. 1999. Measurement of the ability in monocular depth perception during gazing at near visual target-effect of the ocular parallax cue. In Proc. IEEE International Conference on Systems, Man, and Cybernetics, Vol. 2. 34-37.

Michael F. Land. 1995. Fast-focus telephoto eye. Nature 373 (1995), 658-659.

Douglas Lanman and David Luebke. 2013. Near-eye light field displays. ACM Trans. Graph. (SIGGRAPH Asia) 32, 6 (2013), 220:1-220:10.

Sheng Liu, Dewen Cheng, and Hong Hua. 2008. An optical see-through head mounted display with addressable focal planes. In Proc. ISMAR. 33-42.

Patrick Llull, Noah Bedard, Wanmin Wu, Ivana Tošić, Kathrin Berkner, and Nikhil Balram. 2015. Design and optimization of a near-eye multifocal display system for augmented reality. In Imaging and Applied Optics 2015, JTh3A.5.

Gordon D. Love, David M. Hoffman, Philip J. W. Hands, James Gao, Andrew K. Kirby, and Martin S. Banks. 2009. High-speed switchable lens enables the development of a volumetric stereoscopic display. Opt. Express 17, 18 (Aug. 2009), 15716-15725.

Andrew Maimone, Andreas Georgiou, and Joel S. Kollin. 2017. Holographic near-eye displays for virtual and augmented reality. ACM Trans. Graph. (SIGGRAPH) 36, 4 (2017), 85:1-85:16.

Alistair P. Mapp and Hiroshi Ono. 1986. The rhino-optical phenomenon: Ocular parallax and the visible field beyond the nose. Vision Research 26, 7 (1986), 1163-1165.

Jonathan A. Marshall, Christina A. Burbeck, Dan Ariely, Jannick P. Rolland, and Kevin E. Martin. 1996. Occlusion edge blur: A cue to relative visual depth. F. Opt. Soc. Am. A 13, 4 (1996), 681-688.

George Mather and David R. R. Smith. 2002. Blur discrimination and its relation to blur-mediated depth perception. Perception 31, 10 (2002), 1211-1219.

Michael Mauderer, Simone Conte, Miguel A. Nacenta, and Dhanraj Vishwanath. 2014 Depth perception with gaze-contingent depth of field. (2014), 217-226.

Suzanne P. Mckee and Ken Nakayama. 1984. The detection of motion in the peripheral visual field. Vision Research 24, 1 (1984), 25-32.

Olivier Mercier, Yusufu Sulai, Kevin Mackenzie, Marina Zannoli, James Hillis, Derek Nowrouzezahrai, and Douglas Lanman. 2017. Fast gaze-contingent optimal decompositions for multifocal displays. ACM Trans. Graph. (SIGGRAPH Asia) 36, 6 (2017).

Phillip E. Napieralski, Bliss M. Altenhoff, Jeffrey W. Bertrand, Lindsay O. Long, Sabarish V. Babu, Christopher C. Pagano, Justin Kern, and Timothy A. Davis. 2011 Near-field distance perception in real and virtual environments using both verbal and action responses. ACM Trans. Appl. Percept. 8, 3, Article 18 (Aug. 2011), 19 pages.

R. Narain, R. Albert, A. Bulbul, G. J. Ward, M. S. Banks, and J. F. O’Brien. 2015. Optimal presentation of imagery with focus cues on multi-plane displays. ACM Trans. Graph. (SIGGRAPH) 34, 4 (2015).

Mark Nawrot. 2003. Eye movements provide the extra-retinal signal required for the perception of depth from motion parallax. Vision Research 43, 14 (2003), 15531562.

Kenneth N. Ogle and J. Theodore Schwartz. 1959. Depth of focus of the human eye. JOSA 49, 3 (1959), 273-280.

Nitish Padmanaban, Robert Konrad, Tal Stramer, Emily A. Cooper, and Gordon Wetzstein. 2017. Optimizing virtual reality for all users through gaze-contingent and adaptive focus displays. PNAS 114, 9 (2017), 2183-2188.

Nitish Padmanaban, Yifan Peng, and Gordon Wetzstein. 2019. Holographic near-eye displays based on overlap-add stereograms. ACM Trans. Graph. 38, 6, Article 214 (Nov. 2019), 13 pages. DOI : https://doi.org/10.1145/3355089.3356517

Stephen E. Palmer. 1999. Vision Science-Photons to Phenomenology. MIT Press.

Stephen E. Palmer and Joseph L. Brooks. 2008. Edge-region grouping in figure-ground organization and depth perception. f. Exp. Psychol. Hum. Percept. Perform. 34, 6 (2008), 1353.

Anjul Patney, Marco Salvi, Joohwan Kim, Anton Kaplanyan, Chris Wyman, Nir Benty, David Luebke, and Aaron Lefohn. 2016. Towards foveated rendering for gazetracked virtual reality. ACM Trans. Graph. (SIGGRAPH Asia) 35, 6 (2016), 179:1179:12.

John D. Pettigrew, Shaun P. Collin, and Matthias Ott. 1999. Convergence of specialised behaviour, eye movements and visual optics in the sandlance (Teleostei) and the chameleon (Reptilia). Curr. Biol. 9, 8 (1999), 421-424.

Rebekka S. Renner, Boris M. Velichkovsky, and Jens R. Helmert. 2013. The perception of egocentric distances in virtual environments-A review. ACM Comput. Surv. 46, 2 (2013), 23:1-23:40.

Jannick P. Rolland, Myron W. Krueger, and Alexei Goon. 2000. Multifocal planes headmounted displays. OSA Appl. Opt. 39, 19 (2000), 3209-3215.

Heiko H. Schütt, Stefan Harmeling, Jakob H. Macke, and Felix A. Wichmann. 2016 Painfree and accurate Bayesian estimation of psychometric functions for (potentially) overdispersed data. Vision Research 122 (2016), 105-123.

Peter Shirley, Michael Ashikhmin, and Steve Marschner. 2009. Fundamentals of Computer Graphics. A. K. Peters/CRC Press.

Andrew T. Smith and Robert J. Snowden. 1994. Visual Detection of Motion. Academic Press. Retrieved from https://books.google.com/books?id=p11qAAAAMAAJ.

Dhanraj Vishwanath and Erik Blaser. 2010. Retinal blur and the perception of egocentric distance. 7. Vision 10, 10 (Aug. 2010), 26-26.

Gerald Westheimer. 1954. Eye movement responses to a horizontally moving visual stimulus. A.M.A. Arch. Ophthalmol. 52, 6 (1954), 932-941.

Felix A. Wichmann and N. Jeremy Hill. 2001a. The psychometric function: I. Fitting, sampling, and goodness of fit. Percept. Psychophys. 63, 8 (1 Nov. 2001), 1293-1313.

Felix A. Wichmann and N. Jeremy Hill. 2001b. The psychometric function: II. Bootstrap-based confidence intervals and sampling. Percept. Psychophys. 63, 8 (1 Nov. 2001), 1314-1329.

Albert Yonas, Lincoln G. Craton, and William B. Thompson. 1987. Relative motion: Kinetic information for the order of depth at an edge. Percept. Psychophys. 41, 1 (1 Jan. 1987), 53-59.

Marina Zannoli, Rachel A. Albert, Abdullah Bulbul, Rahul Narain, James F. O’Brien, and Martin S. Banks. 2014. Correct blur and accommodation information is a reliable cue to depth ordering. F. Vision 14, 10 (2014), 138.

Marina Zannoli, Gordon D. Love, Rahul Narain, and Martin S. Banks. 2016. Blur and the perception of depth at occlusions. F. Vision 16, 6 (Apr. 2016), 17-17.

Received June 2019; revised September 2019; accepted October 2019 